\title{
Hybrid mobile power supply system of Multimedia Hybrid Mobile Stages
}

\author{
Valeriy Martynyuk ${ }^{1 *}$, Tomasz Kałaczyński ${ }^{2}$, Roman Petrus $^{3}$ and Hryhoriy Ilchuk $^{3}$ \\ ${ }^{1}$ Khmelnytsky National University, 29016, 11 Institutska Street, Khmelnytsky, Ukraine \\ ${ }^{2}$ Bydgoszcz University of Science and Technology, Faculty of Mechanical Engineering, Kaliskiego \\ Street 7, 85-796 Bydgoszcz, Poland \\ ${ }^{3}$ Lviv Polytechnic National University, 79013, 12 Bandera Street, Lviv, Ukraine
}

\begin{abstract}
You should leave $8 \mathrm{~mm}$ of space above the abstract and $10 \mathrm{~mm}$ after the abstract. The heading Abstract should be typed in bold 9-point Arial. The body of the abstract should be typed in normal 9-point Times in a single paragraph, immediately following the heading. The text should be set to 1 line spacing. The abstract should be centred across the page, indented $17 \mathrm{~mm}$ from the left and right page margins and justified. It should not normally exceed 200 words.
\end{abstract}

\section{Introduction}

Multimedia Hybrid Mobile Stage (MHMS) is a folding concert complex based on a trailer. It has a standard stage design that converts to smaller dimensions for transportation. MHMS is intended for holding concert events in the absence of stationary concert venues. The MHMS is usually a bar structure, the main components of which are reduced to beams and columns shaped as trusses, flat or spatial frames as shown in Figure 1 [1].

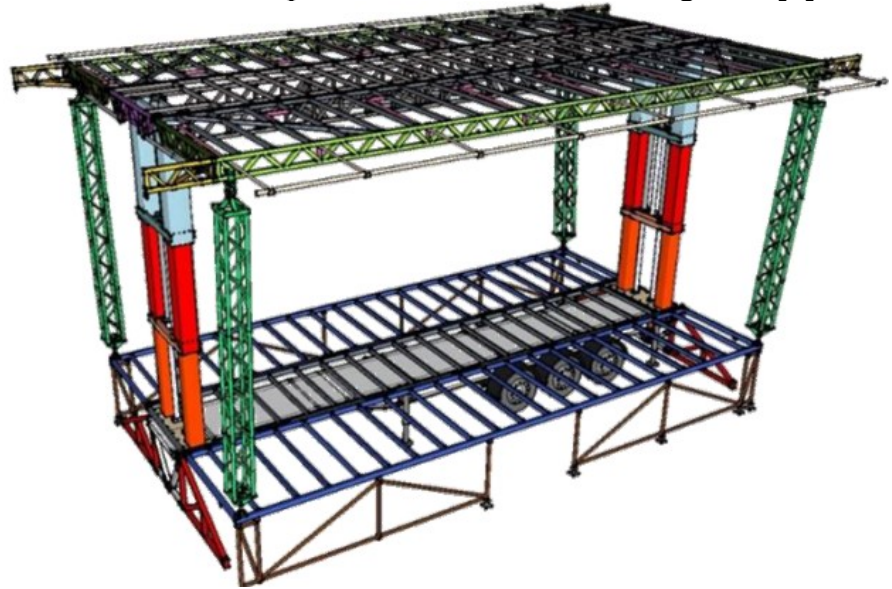

* Corresponding author: martynyuk.valeriy@gmail.com 
Fig. 1. Multimedia Hybrid Mobile Stage.

The power supply of MHMS is based on a diesel generator or a gasoline electric generator. It is possible to increase the efficiency of the MHMS power supply system by means of the Hybrid Mobile Power Supply System (HMPSS).

Today, autonomous energy supply systems using renewable energy sources for use in different geographical parts of the world are being intensively studied, both theoretically and experimentally [2] - [5]. However, for remote regions, where the implementation of the central grid is not economically feasible, the best option is to use HMPSS, which contain several sources of electricity generation: usually renewable photovoltaic modules (PM), and traditional - usually diesel generator (DG) [3], [4].

Among the main reasons that motivate scientists around the world to such studies are the reduction of greenhouse gas emissions through the use of renewable energy sources [2], [6]; ensuring the possibility of power supply to remote regions, taking into account economic (related to the cost of diesel fuel) aspects, reliability and environmental pollution [5] - [7]; providing electricity to rural areas of underdeveloped countries [7]; a reliable source of energy for rapid response teams in remote disaster areas to power a vaccine refrigerator, water purification system and basic satellite communication system [8], which has great potential to save lives.

The publications analyze the performance of HMPSS taking into account the average hourly insolation of sunlight, ambient temperature and load power profile [3]. Mathematical models are being developed that can be used to estimate the size, control and performance of HMAPSS, which contains photovoltaic modules (PM), diesel generator (DG) and rechargeable accumulator battery $(\mathrm{AB})$ and supercapacitor (SCB) battery [3], [5]. In [6], the behavior of $\mathrm{AB}$ under the influence of alternating $\mathrm{PM}$ lighting is studied, and DG is used as a backup energy source when the PM power is insufficient.

In the literature, mainly studies of low-power HMPSS (up to several $\mathrm{kW}$ ), which have both DC and AC output voltage [7], [8]. There are almost no studies of processes and methods to increase the efficiency of energy extraction from PM, compensation of peak (starting) loads, balancing, control of operating temperature and multi-stage charge of the battery for the longevity of their operation. All these scientific problems require in-depth research.

\section{HMPSS mathematical model}

HMPSS mathematical model combines four scientific and applied techniques. The energy from HMPSS can be used in the form of direct or alternating current using appropriate $\mathrm{DC} / \mathrm{DC}$ converters and DC/AC inverter. In particular:

1. The method of two-coordinate tracking of the trajectory of the sun with dynamic positioning of the working surface of the PM perpendicular to the solar radiation and the corresponding control of the tracker in fog, cloudiness, heavy rainfall and strong wind. The use of a dynamic tracking system allows increasing system performance by up to $30 \%$ and reducing the area occupied by the PM.

2. The method of harvesting of electric energy from PM with the use of SCB in comparison with analogues works effectively even in rapidly changing weather conditions and monitors the MPP of each module with sufficient accuracy. In the vast majority of analogues $[2,3]$, the search for the maximum power point (MPP) is carried out at the level of sequential PM string. However, it is known that in a string, all solar panels cannot work in the MPP if it is formed by a direct electrical connection of the PM.

3. The charge-discharge method of the accumulator batteries (AB) and SCB according to the developed algorithm that provides minimal energy losses on voltage converters, multi-stage charge mode, control of temperature and voltage of $\mathrm{AB}$ in order to increase their service life. 
An additional advantage of using SCB (over existing analogues) in HMPSS is the ability to obtain high instantaneous output power even at low insolation of solar radiation (power amplification effect) due to its low internal resistance and, accordingly, low charging and high discharge current.

4. The method of active balancing of $\mathrm{AB}$ and $\mathrm{SCB}$, the essence of which is to transfer energy from a more charged cell to a less charged cell with almost no energy loss. Equalization of the charge level is a method that allows increasing the safety of battery operation, operating time without recharging and servicing life.

As inverter is a source of alternate voltage for HMPSS with chaotic load. AB and SCB are the sources of currents which may have positive and negative values depending on its charge and discharge modes.

$$
i(t)=i_{L}(\mathrm{t})+i_{0}(\mathrm{t})
$$

where the current $i_{0}(\mathrm{t})$ corresponds to the process of energy transition from AB and SCB to the load, and current $i_{L}(\mathrm{t})$ is the load current under condition $i_{0}(\mathrm{t})=0$.

To increase the efficiency of HMPSS it is necessary to fulfill the condition of average current $i_{L}(\mathrm{t})$ minimization at stable voltage $u(\mathrm{t})$ amplitude. In this case, current $i_{L}(\mathrm{t})$ cannot exceed the threshold value, after which the source suffers voltage decrease, meanwhile energy consumption from the source must be in its maximum. Thus, the condition may be described by the following expression:

$$
\left\{\begin{array}{l}
A_{\min } \sin \left(\omega t+\varphi_{0}\right)<\mathrm{u}(\mathrm{t})<A_{\max } \sin \left(\omega t+\varphi_{0}\right) \\
u(t) \rightarrow A_{0} \sin \left(\omega t+\varphi_{0}\right) \\
i(t)=i_{L}(t)+i_{0} \\
\sqrt{\frac{1}{T} \int_{0}^{T}\left(i_{P V}(\tau) \cdot u_{P V}(\tau)\right)^{2} d \tau} \rightarrow \max
\end{array}\right.
$$

Fulfillment of this condition is provided by the algorithm of current control, while the current is supplied from the energy in AB and SCB $i_{0}(\mathrm{t})$. The block diagram of HMPSS with the possibility to realize virtual impedance is shown in Figure 2.

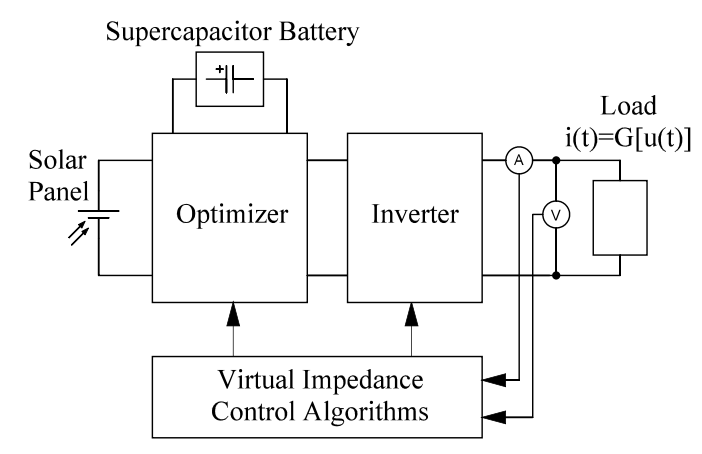

Fig. 2. Block diagram of HMPSS.

The simulation model of HMPSS based on AB and SCB is shown in Figure 3. 


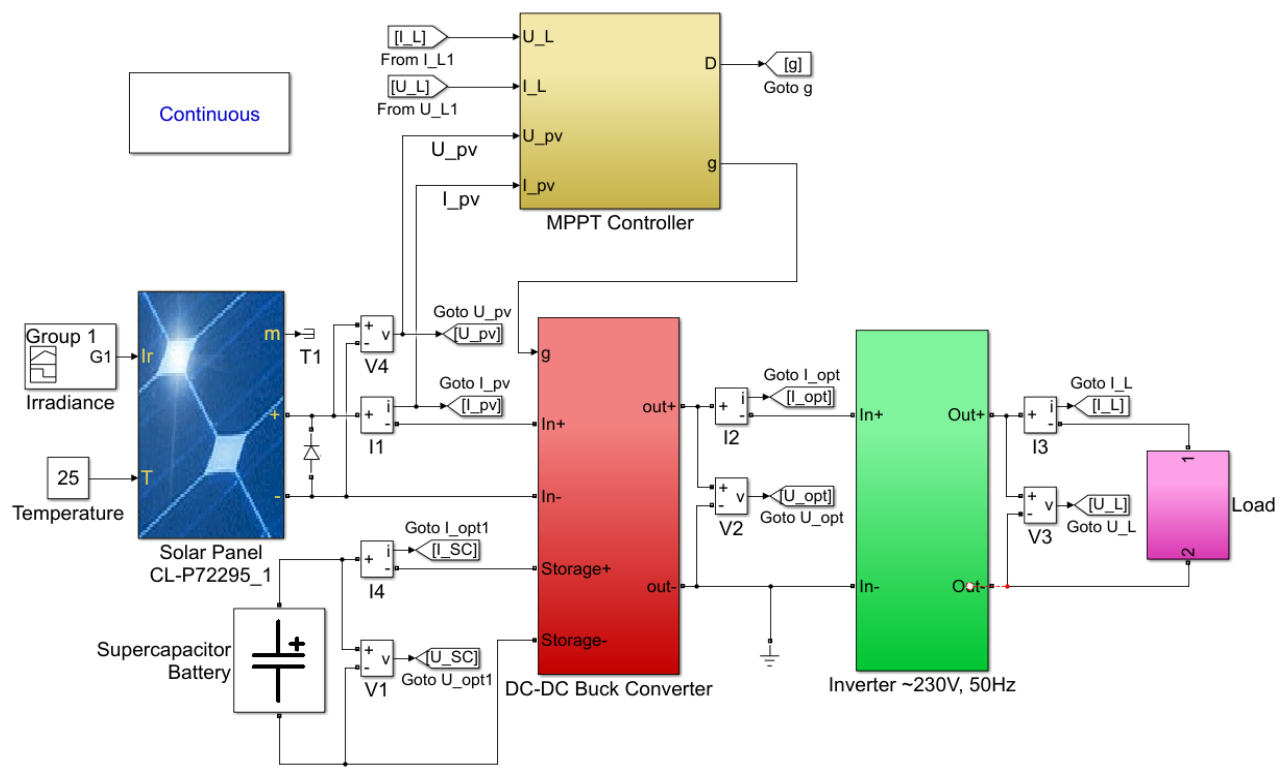

Fig. 3. Block diagram of HMPSS.

The presented simulation model gives the possibility to increase the efficiency of the developed HMPSS on PM, AB and SCB by broadening the scopes of transient process analysis at the unstable nonlinear load. The model enables direct setting of the algorithm for PVM maximum power point tracking in the form of MATLAB function, which may be converted to binary code for the controller of the DC-DC converter.

The DC-DC converter is shown in Figure 4. The DC-DC converter is part of the solar panel optimizer, which is designed to track of the maximum power point, and thus increase the efficiency of energy transfer to the load from the solar panel.

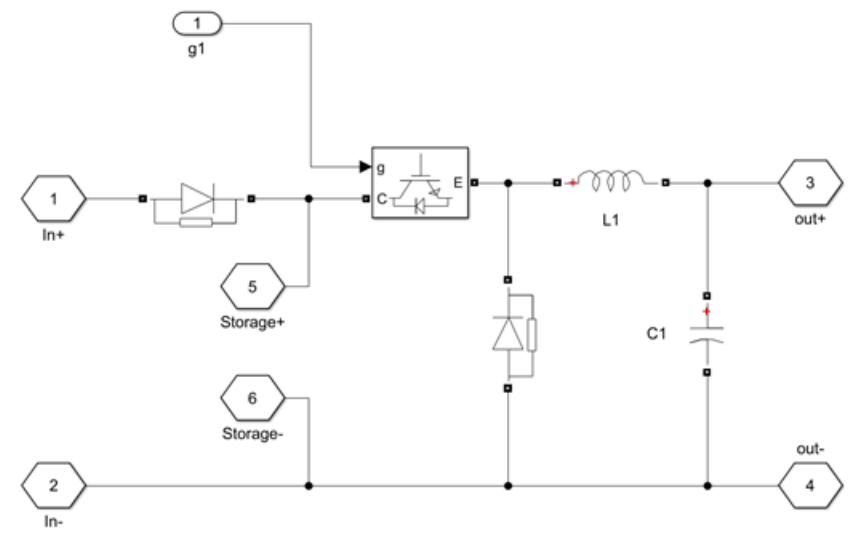

Fig. 4. Block diagram of DC-DC converter.

The DC-DC converter is built on a transistor switch. The DC-DC converter consists of two protective diodes, as well as an output filter, which is built on the inductor L1 and capacitor $\mathrm{C} 1$. The control signal $\mathrm{g}$ is designed to control the DC-DC converter. This signal is generated by the MPPT controller. The simulation model of the MPPT controller is shown in Figure 5. 


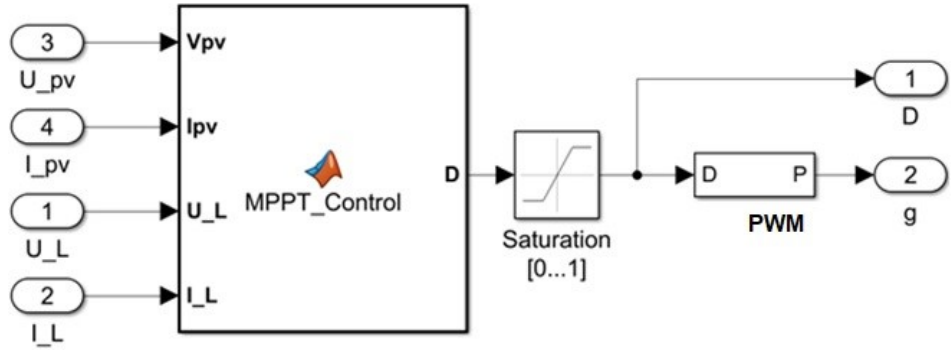

Fig. 5. Block diagram of MPPT controller.

The MPPT control algorithm has been developed as a function in the MATLAB simulation environment. The MPPT controller receives input data in the form of current and voltage of the solar panel, as well as the load.

After determining the operating point of the solar panel and the value of its compliance with the load resistance, the control signal is formed. The control signal of the MPPT controller is fed to the PWM signal generator. The PWM signal generator opens and closes the transistor switch of the DC-DC converter.

From the output of the DC-DC converter voltage is supplied to the inverter input. The simulation model of the inverter is shown in Figure 6.

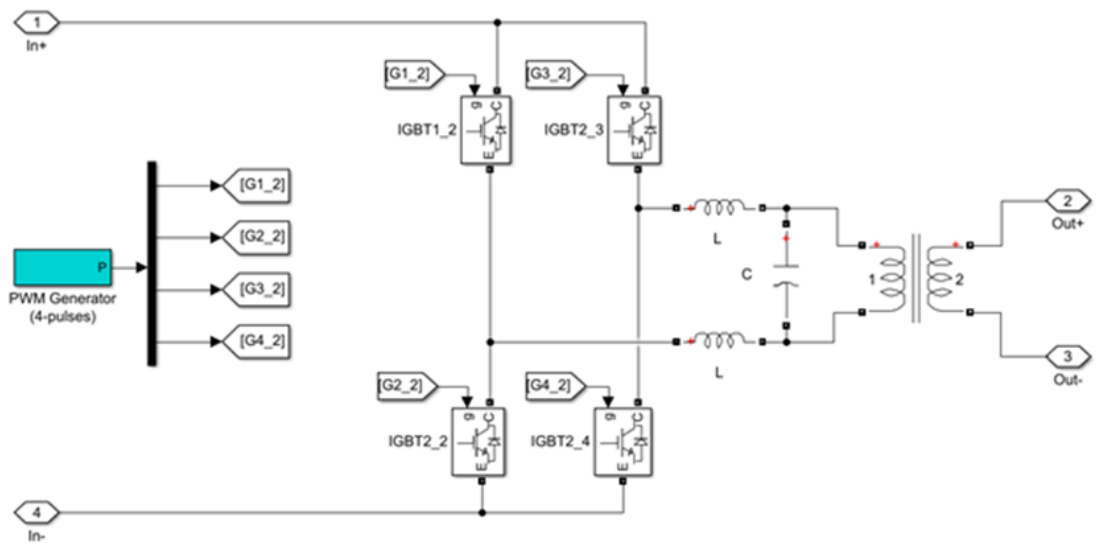

Fig. 6. Block diagram of inverter.

The inverter converts the voltage supplied from the DC-DC converter into $220 \mathrm{~V}$ AC voltage. The inverter generates an $\mathrm{AC}$ voltage with a frequency of $50 \mathrm{~Hz}$. The inverter is implemented using a bridge circuit. A feature of the bridge circuit is the use of four transistor switches, which are implemented on IGBT transistors.

The PWM signal generator supplies control signals to the IGBT transistors. After the inverter, the low-pass filter is switched on. The low-pass filter smoothes the output voltage and the transformer. The transformer generates a voltage of $325.2 \mathrm{~V}$ at the output of the DCDC converter.

To increase the energy from the solar panel, it is necessary to change the transfer function of the inverter over time in order to change the equivalent linear part of the load circuit to obtain the maximum effect. 
The phenomenon of nonlinearity of the load occurs due to the presence in the network of nonlinear elements, such as rectifiers, voltage converters, magnetic circuits of transformers, as well as due to switching of electricity consumers.

In power grids with nonlinear load, current fluctuations can have chaotic dynamics, which is determined by the characteristics of nonlinear elements connected to the network.

The presence in the network of such elements as, for example, rectifiers or voltage converters, built on the basis of semiconductor diodes, can lead to current fluctuations at frequencies other than the nominal one. Such oscillations are usually characterized by an unstable offset relative to the midpoint, and correspond to the oscillations generated by systems with multistable potential functions.

The simplest block diagram of the load, which has chaotic modes of current fluctuations, is shown in Figure 7.

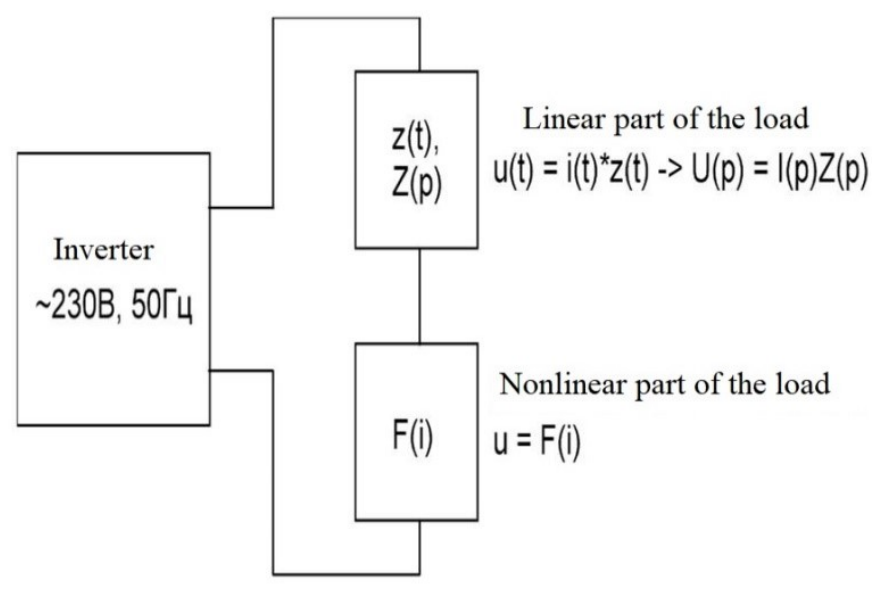

Fig. 7. The simplest block diagram of the load.

Most loads with chaotic dynamics can be described by a type equation, which determines the dependence of the voltage from current at the load.

$$
\mathrm{A}(t) *\left(i(\mathrm{t})+\mathrm{F}\left[i(t), i^{\prime}(t), i^{\prime \prime}(t), \ldots, i^{(n)}(t)\right]\right)=u(t) .
$$

The voltage of the inverter is the master oscillation for a chaotic system formed by the load. The current flowing through the terminals of the inverter depends on the voltage of the inverter, is the initial value of the chaotic system. A chaotic load system consists of linear and nonlinear parts.

The linear part is described by the impulse response $\mathrm{A}(t)$. The convolution of current $i(t)$ with a pulse characteristic $\mathrm{A}(t)$ determines the equivalent voltage of the linear part of the chaotic system:

$$
\mathrm{u}_{L}(\mathrm{t})=\mathrm{A}(t) * i(\mathrm{t})=\int_{0}^{t} A(\tau) i(t-\tau) d \tau
$$

The nonlinear part of a chaotic system is described by a nonlinear function of the current at the terminals of the inverter and its derivatives from the first to the $n$-th order. The equivalent voltage of the nonlinear part of the chaotic system is determined by the expression: 


$$
\mathrm{u}_{N L}(\mathrm{t})=\mathrm{A}(t) * \mathrm{~F}\left[i(t), i^{\prime}(t), i^{\prime \prime}(t), \ldots, i^{(n)}(t)\right] .
$$

The sum of the equivalent voltages of the linear and nonlinear parts is the value of the master voltage fluctuation at the output of the inverter:

$$
u(t)=u_{L}(t)+u_{N L}(t)
$$

Then the equation of the chaotic load model has the form:

$$
\mathrm{A}(t) *\left(i_{L}(\mathrm{t})+i_{0}(\mathrm{t})+\mathrm{F}\left[i_{L}(\mathrm{t})+i_{0}(\mathrm{t}), i_{L}{ }^{\prime}(\mathrm{t}), i_{L}{ }^{\prime \prime}(\mathrm{t}), \ldots, i_{H}{ }^{(n)}(\mathrm{t})\right]\right)=u(t)
$$

The block diagram of chaotic load is shown in Figure 8.

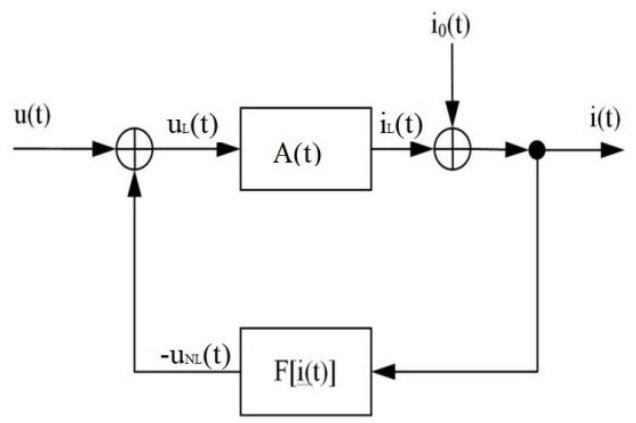

Fig. 8. The block diagram of the chaotic load.

The mathematical model of the chaotic load, shown in Figure 8 , is described by the Duffing equation with coefficients that provide scaling in time and amplitude:

$$
B_{1} \cdot \frac{d^{2} i(t)}{d t^{2}}+B_{2} \cdot k \cdot \frac{d i(t)}{d t}+B_{3} \cdot F\left(B_{4} \cdot i(t)\right)=u(t),
$$

where $B_{1}$ and $B_{2}$ are the frequency scaling coefficients;

$$
B_{1}=\frac{1}{\omega^{2}} \text { and } B_{2}=\frac{1}{\omega},
$$

where $\omega=2 \cdot \pi \cdot f$ is an angular frequency;

$k$ is attenuation coefficient;

$F(x)=x^{3}-x$ is the nonlinear function;

$B_{3}$ and $B_{4}$ are the voltage scaling coefficients;

$$
B_{3}=\frac{U_{0}}{U_{p}} \text { and } B_{4}=\frac{U_{p}}{U_{0}},
$$

where $U_{0}=325,2 \mathrm{~V}$ is a voltage amplitude in the network;

$U_{s}$ is the amplitude of the voltage that corresponds to the set mode of oscillation

The simulation model of load with chaotic dynamics is shown in the Figure 9. 


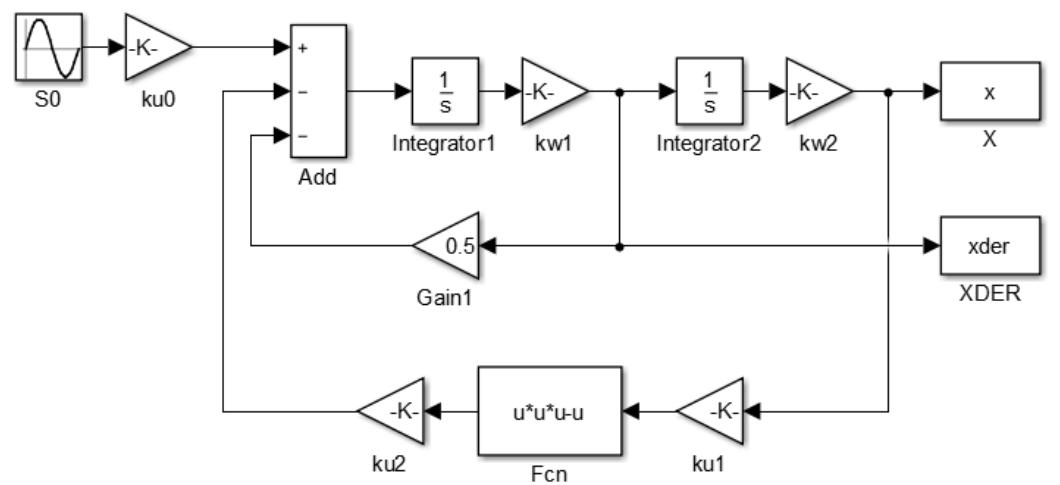

Fig. 9. Simulation model of the nonlinear unstable load, which is characterized by the dynamics of the Duffing system.

In the given simulation model the values of coefficients which correspond to scaling of equation (8) for work in a network of alternating voltage of $\sim 230 \mathrm{~B}$ with a frequency of 50 $\mathrm{Hz}$ are established.

The coefficients $B_{1}$ and $B_{2}$ for the $50 \mathrm{~Hz}$ network frequency have fixed values:

$$
B_{1}=1,013 \cdot 10^{-5} \text { and } B_{2}=0.0032
$$

Coefficients $B_{3}$ and $B_{4}$ for both mains voltage $230 \mathrm{~V}$ and range $U_{s} \in[0,3 ; 0,9](\mathrm{V})$ are determined from the conditions:

$$
B_{3} \in[361 ; 1000] \text { and } B_{4}=\frac{1}{B_{3}}
$$

Coefficients $B_{3}$ and $B_{4}$ determine the mode of the load current. In the simulation model shown in Figure 9 coefficients have the following values:

$$
\begin{gathered}
k w 1=\omega=314,2 \text { and } k w 2=k w 1=\omega=314,2 . \\
k u 2=B_{3}=325,2 / U_{p}, k u 1=B_{4}=U_{p} / 325,2 \text { and } k u 0=1 .
\end{gathered}
$$

The voltage and current of the load with the chaotic dynamics described by the Duffing system are given in Figure 10. 


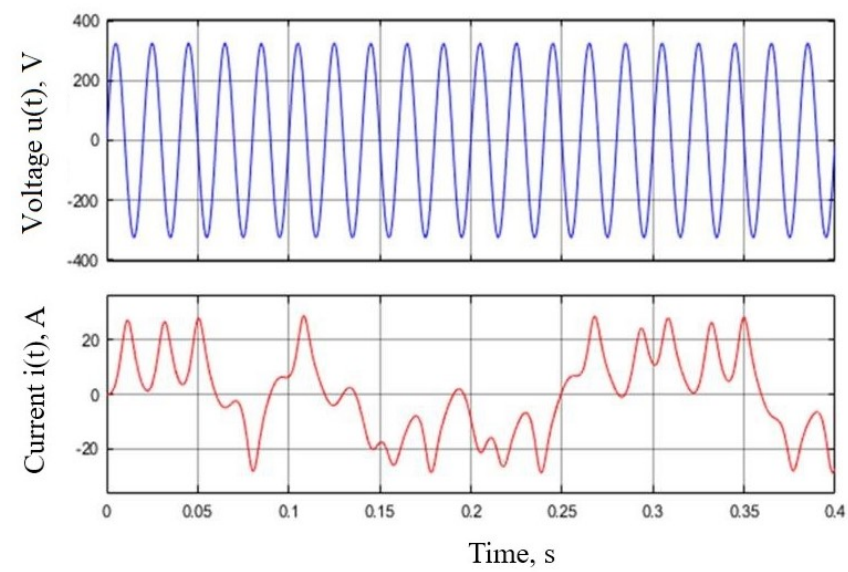

Fig. 10. Voltage and current of the load with chaotic dynamics in the Simulink.

The surface formed by the instantaneous values of the current on the load, which is described by equation (8) and depending on the time and voltage in the network is shown in Figure 11 .

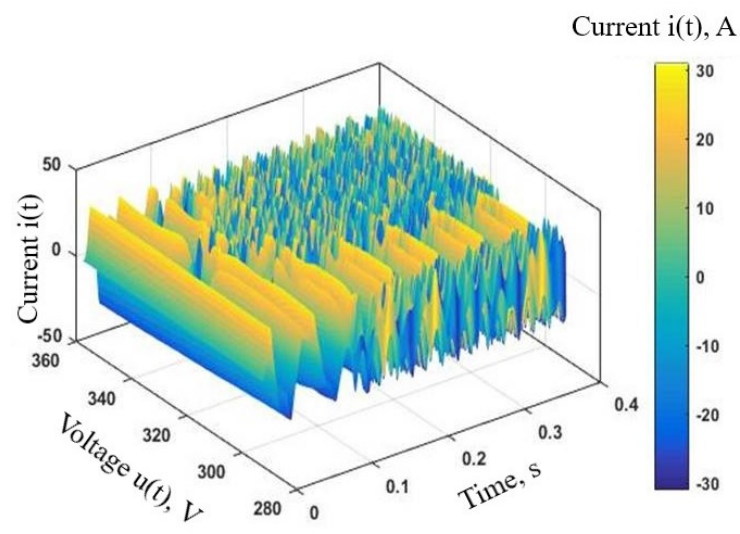

Fig. 11. Dependence of the chaotic load current on voltage over time.

As shown in Figure 4.11, the current of the nonlinear unstable load significantly depends on small voltage changes in the network.

\section{Conclusions}

The proposed Hybrid Mobile Power Supply System contains photovoltaic modules, rechargeable accumulator battery and supercapacitor battery allows to minimize negative influence of nonlinear unstable dynamics of the current load by means of the maximum power point tracking.

According to the maximum power tracking algorithm and the energy storage based on a battery of supercapacitors, which is characterized by large peak currents, the impact of 
chaotic oscillations of unstable load on the instantaneous power of the solar panel is minimized.

The fluctuations in the instantaneous power of the solar panel in time are caused mainly by means of the optimizer, which tracks the maximum power point according to the perturb and observation algorithm.

The proposed system of compensation allows to significantly increase the efficiency of the solar panel by minimizing the dependence of the solar panel power on the load instability using the maximum power point algorithm taking into account the characteristics of the rechargeable accumulator battery and supercapacitor battery.

The given simulation model allows to simplify the process of developing the Hybrid Mobile Power Supply System by automated calculation of parameters, characteristics and modes of operation of its moduled under different operating conditions of the solar panel and unstable mains inverter loads.

This paper has been achieved under the research project "Hybrid multimedia mobile scenes are a chance for decisive innovation" No. POIR.04.01.04-00-0045/17-00

\section{References}

1. M. Liss,, T. Kałaczyński, N. Dluhunovych, A. Dykha and R. Martinod Identification of loads of the construction of a Hybrid Multimedia Mobile Stage, $19^{\text {th }}$ International Conference Diagnostics of Machines and Vehicles, MATEC Web of Conferences, 332, (2021)

2. B. Brahim Performance investigation of a hybrid PV-diesel power system for remote areas, Int. J. Energy Res., vol. 43, no. 2, pp. 1019-1031, Feb. 2019.

3. The Energy Cost Analysis of Hybrid Systems and Diesel Generators in Powering Selected Base Transceiver Station Locations in Nigeria, Energies, vol. 11, no. 3, p. 687, Mar. 2018.

4. A. Nayak, K. Kasturi, and M. R. Nayak Cycle-charging dispatch strategy based performance analysis for standalone PV system with DG \&amp BESS, in 2018 Technologies for Smart-City Energy Security and Power (ICSESP), 2018, pp. 1-5.

5. A. Maleki Modeling and optimum design of an off-grid PV/WT/FC/diesel hybrid system considering different fuel prices, Int. J. Low-Carbon Technol., vol. 13, no. 2, pp. 140147, Jun. 2018.

6. P. Tripathi, M. A. Ansari, M. J. Khan, and S. Yadav Modelling of Energy Efficient PVDiesel-Battery Hybrid system in 2018 International Conference on Computational and Characterization Techniques in Engineering \& Sciences (CCTES), 2018, pp. 1-5.

7. C.-T. Tsai, T.-W. Shen, Y.-P. Chen, and P.-H. Hsu Control Strategy of PV/Diesel/Battery Hybrid System for Island-Based Microgrid in 2018 International Symposium on Computer, Consumer and Control (IS3C), 2018, pp. 121-124.

8. A. Khan and R. Khan Cost Optimization of Hybrid Microgrid using Solar PV, Fuel Cell and Diesel Generator in HOMER in $20182^{\text {nd }}$ International Conference on Energy Conservation and Efficiency (ICECE), 2018, pp. 14-18. 